\title{
HIDRÓXIDOS DUPLOS LAMELARES À BASE DE ESCÓRIA DE ALTO FORNO
}

\author{
Monaliza Maia Rebelo*, Márcia Valéria Porto de Oliveira Cunha e José Augusto Martins Corrêa \\ Instituto de Geociências, Universidade Federal do Pará, Av. Augusto Corrêa, 1, 66075-110 Belém - PA, Brasil
}

Recebido em 14/4/11; aceito em 8/11/11; publicado na web em 13/1/12

\begin{abstract}
LAYERED DOUBLE HYDROXIDES PRODUCED FROM BLAST FURNACE SLAG. Hydrotalcite like compounds (HT) were prepared by co-precipitation $(\mathrm{Mg} / \mathrm{Al}=3.5)$, using an acid solution with blast furnace slag and $\mathrm{MgCl}_{2} \cdot 6 \mathrm{H}_{2} \mathrm{O}$ and aqueous solutions of $\mathrm{NaOH}$. The following synthesis variables were investigated: temperature $\left(30\right.$ and $\left.45^{\circ} \mathrm{C}\right)$ and $\mathrm{pH}(9$ and 12). Depending of the temperature two systems were observed: $\mathrm{Mg}-\mathrm{Al}-\mathrm{CO}_{3}\left(\mathrm{~T}=30^{\circ} \mathrm{C}\right) ; \mathrm{Mg}-\mathrm{Al}-\mathrm{Cl}-\mathrm{CO}_{3}\left(\mathrm{~T}=45^{\circ} \mathrm{C}\right)$. An increase in the $\mathrm{pH}$ of synthesis and $\mathrm{Mg}^{2+}$ concentration produced HTs well-crystallized and with greater values of all cell parameters. The study showed the potentiality of BFS in the synthesis of well-crystallized LDHs without the presence of other crystalline phases.
\end{abstract}

Keyords: blast furnace slag; layered double hydroxides (LDHs); syntheses.

\section{INTRODUÇÃO}

A atual situação em torno da disposição de resíduos industriais tornou-se uma séria preocupação que ameaça o meio ambiente, a saúde e a qualidade de vida. Quando os resíduos industriais são dispostos de maneira inadequada, causam graves problemas ambientais como contaminação das águas subterrâneas por lixiviação de metais tóxicos e emissões de material particulado para áreas vizinhas. A reciclagem de resíduos mostra-se como uma alternativa capaz de minimizar os impactos ambientais negativos decorrentes do processo produtivo. ${ }^{1}$

A produção de aço gera uma série de resíduos industriais. Entre os vários tipos de resíduos, a escória de alto forno (EAF) tem atraído muita atenção, já que a sua produção ultrapassa a de qualquer outro resíduo. ${ }^{1}$ A indústria do aço no Brasil, representada por 14 empresas privadas, foi responsável pela produção, em 2010, de 32,9 milhões de toneladas de aço bruto, levando o país a ocupar a $9^{\text {a }}$ posição no ranking da produção mundial. ${ }^{2}$ De acordo com análises do Instituto Aço Brasil, a produção acumulada de janeiro a novembro de 2011 totalizou 34,9 milhões de toneladas de aço bruto e 27,3 milhões de toneladas de ferro-gusa, o que representa, em média, um volume total de 13,4 milhões de toneladas de escórias, oriundas do alto forno e da aciaria. ${ }^{2}$

A EAF é formada pela mistura entre a ganga do minério de ferro com as cinzas do coque ou carvão vegetal e do calcário, utilizado como fundente no processo de fabricação do ferro-gusa. ${ }^{3}$ Sua composição química é majoritariamente $\mathrm{Ca}, \mathrm{Si}, \mathrm{Al}$ e $\mathrm{Mg}$, bem como uma pequena quantidade de $\mathrm{Fe}$, Ti e $\mathrm{Mn} .^{3-5} \mathrm{O}$ tamanho de suas partículas e estrutura variam em função da velocidade de resfriamento da EAF na água ou no ar. Ao ser resfriada bruscamente, a EAF adquire estrutura vítrea e forma granular, apresentando propriedades hidráulicas latentes; mas, se o processo empregado for o resfriamento lento, a escória cristaliza, formando um produto sem atividade hidráulica, constituído por silicatos e aluminossilicatos cálcicos. Nas escórias básicas $\left(\mathrm{CaO} / \mathrm{SiO}_{2}>1\right)$ há predominância de melilitas (akermanita e gehlenita) e merwinita, enquanto que nas ácidas $\left(\mathrm{CaO} / \mathrm{SiO}_{2}<1\right)$ ocorre principalmente wollastonita e anortita. ${ }^{4,6,7}$

A EAF é um material nobre por suas características físico-químicas que, moída a uma granulometria adequada e com adição de ativadores, adquire propriedades aglomerantes. ${ }^{6} \mathrm{~A}$ EAF tem sido reciclada principalmente como cimento hidráulico, concreto, agregado e materiais de pavimentação em trabalhos de engenharia civil. ${ }^{8,9}$ No

*e-mail: monalizamaia@yahoo.com.br entanto, a produção de ferro e, consequentemente, de EAF aumentou em todo o mundo, de tal forma que o volume de EAF produzido, em breve, ultrapassará a demanda e o consumo. É essencial, portanto, o contínuo desenvolvimento de processos de reciclagem de EAF novos e avançados.

O uso da EAF como produto de partida para a síntese de HDLs deve-se às suas características químicas, especialmente à presença de metais di e trivalentes, em particular o alumínio.

Os HDLs, também conhecidos como compostos do tipo hidrotalcita, possuem uma fórmula geral que é representada por $\mathrm{M}^{2+}{ }_{(1-\mathrm{x})}$ $\mathrm{M}^{3+}{ }_{\mathrm{x}}(\mathrm{OH})_{2}\left(\mathrm{~A}^{\mathrm{m}-}\right)_{\mathrm{x} / \mathrm{m}} \cdot \mathrm{nH}_{2} \mathrm{O}$ (abreviado como $\left.\mathrm{M}^{2+}-\mathrm{M}^{3+}-\mathrm{A}^{\mathrm{m}-}\right)$, onde $\mathrm{M}^{2+}$ e $\mathrm{M}^{3+}$ são íons metálicos di e trivalentes, respectivamente, $\mathrm{A}^{\mathrm{m}-}$ é um ânion intercalado, $\mathrm{x}$ é a fração molar do cátion $\mathrm{M}^{3+}\left[\mathrm{x}=\mathrm{M}^{3+}\right.$ / $\left.\left(\mathrm{M}^{2+}+\mathrm{M}^{3+}\right)\right]$ e $\mathrm{n}$ é o número de moléculas de água. ${ }^{10,11}$ São compostos que apresentam características estruturais onde cátions metálicos, geralmente, di e trivalentes, ocupam o interior de octaedros e estão ligados com íons hidroxila nos seus vértices, organizados em uma estrutura semelhante à da brucita $\left(\mathrm{Mg}(\mathrm{OH})_{2}\right)$. Quando cátions divalentes são isomorficamente substituídos por trivalentes, gera-se um excesso de cargas positivas nas lamelas que é compensado por ânions interlamelares e moléculas de água. ${ }^{12}$

Os HDLs vêm recebendo uma crescente atenção por possuírem uma ampla variedade de aplicações, no campo ambiental, medicinal ${ }^{13-15}$ e na indústria de catálise ${ }^{16-18}$ devido a sua: capacidade de troca aniônica; facilidade de acomodação de cátions metálicos na lamela e, elevada basicidade das lamelas superficiais.

A ocorrência natural dos HDLs é bastante limitada. Contudo, sua produção via síntese química pode ser alcançada em laboratório por rotas simples e de baixo custo, o que permite a obtenção de sólidos com elevada pureza de fase e com alto grau de ordem estrutural, com as mais variadas propriedades, ajustadas de acordo com a finalidade desejada. Apesar da expressividade em números de patentes e artigos científicos publicados sobre aplicações de EAF e sobre síntese de HDLs, não se encontra registro algum quanto à aplicação de EAF, como matéria-prima, para a preparação de HDLs com estruturas semelhantes à da hidrotalcita $\left(\mathrm{Mg}-\mathrm{Al}-\mathrm{CO}_{3}\right)$.

Kuwahara et al. ${ }^{1}$ sintetizaram HDLs tipo hidrocalumita (Ca-Al-Cl), usando uma etapa de lixiviação ácida a partir da EAF, seguida de coprecipitação com hidróxido de sódio a $100{ }^{\circ} \mathrm{C}$. Seu produto de síntese mostrou unicamente a presença de hidrocalumita como fase cristalina, cujo grau de ordem estrutural foi maior na medida em que o $\mathrm{pH}$ e a temperatura de síntese foram elevados. As melhores condições 
de síntese para a formação de hidrocalumita foram $\mathrm{pH}(11,5)$, razão molar $\mathrm{Ca} / \mathrm{Al}=2,03$ e principalmente temperatura $\left(100^{\circ} \mathrm{C}\right)$. Foram também observadas quantidades de outros cátions metálicos $(\mathrm{Mg}$, $\mathrm{Fe}, \mathrm{Mn}, \mathrm{Ti}, \mathrm{Si}$ ) na estrutura das hidrocalumitas.

Na patente de Hayaji et al.,${ }^{19}$ os HDLs tipo hidrotalcita (Mg-Al$-\mathrm{CO}_{3}$ ) foram sintetizados a partir de escórias de alumínio e resíduo de cloreto de magnésio líquido. A escória de alumínio foi submetida a um processo de dissolução em diferentes ácidos $\left(\mathrm{HCl}, \mathrm{HNO}_{3}\right.$, $\mathrm{H}_{2} \mathrm{SO}_{4}$ e água régia) à temperatura ambiente. As hidrotalcitas foram formadas em $\mathrm{pH}=10,5$ constante, numa razão molar $\mathrm{Mg} / \mathrm{Al}=2,5$, por gotejamento simultâneo das soluções dos cátions e de $\mathrm{NaOH}$ sobre a solução aquosa de $\mathrm{Na}_{2} \mathrm{CO}_{3}$.

Com base na composição química da EAF e considerando a estequiometria dos HDLs, este trabalho teve como objetivo sintetizar materiais tipo hidrotalcita sem a presença de outras fases cristalinas, por meio da coprecipitação a $\mathrm{pH}$ crescente. Para isso, utilizou-se somente $\mathrm{NaOH}$ como agente precipitante. A EAF foi digerida em ácido clorídrico $(\mathrm{HCl})$ e a solução obtida foi misturada com quantidades suficientes de $\mathrm{MgCl}_{2} \cdot 6 \mathrm{H}_{2} \mathrm{O}$ para estabelecer a razão molar $\mathrm{Mg} / \mathrm{Al}=$ 3,5 . Foram investigados os efeitos da temperatura e do $\mathrm{pH}$ de síntese nas características dos HDLs.

\section{PARTE EXPERIMENTAL}

\section{Fontes de $\mathrm{Al}, \mathrm{Mg}, \mathrm{Cl}$ e $\mathrm{CO}_{3}$}

Como fonte dos elementos utilizados na síntese dos HDLs tipo hidrotalcita foi utilizada escória de alto forno, proveniente de uma siderúrgica da cidade de Marabá/PA ( $\mathrm{Al}, \mathrm{Mg}$ ) e reagentes de pureza analítica $\left(\mathrm{MgCl}_{2} \cdot 6 \mathrm{H}_{2} \mathrm{O}, \mathrm{NaOH}, \mathrm{HCl}\right)$.

\section{Processo de síntese}

A EAF foi pulverizada em um moinho de almofariz, marca Retsch modelo RMO 30903, e em seguida digerida em ácido clorídrico $(\mathrm{HCl})$ e filtrada a vácuo. O resíduo da digestão foi lavado com água deionizada e caracterizado por difração e fluorescência de raios X. A solução ácida obtida foi codificada como solução de EAF e usada para a síntese de HDLs.

OS HDLs foram preparados por coprecipitação a pH crescente. ${ }^{20}$ Foram preparadas duas soluções. A primeira, (solução A), composta da mistura entre a solução de $\mathrm{EAF}$ e $\mathrm{MgCl}_{2} \cdot 6 \mathrm{H}_{2} \mathrm{O}$, com valor teórico da razão molar $\mathrm{Mg} / \mathrm{Al}$ igual a 3,5; a segunda, (solução B), composta somente por $\mathrm{NaOH}$ em meio aquoso.

$\mathrm{O}$ experimento consistiu em misturar as soluções A e B, sob vigorosa agitação. Utilizou-se um peagâmetro digital, marca Schott modelo Handylab 1, para medir o valor de $\mathrm{pH}$ final desejado. A suspensão formada foi agitada por mais $4 \mathrm{~h}$ e, em seguida, o gel de síntese foi submetido a um tratamento hidrotérmico a $100{ }^{\circ} \mathrm{C}$ por 19 $\mathrm{h}$, seguido de filtração, lavagem com água deionizada até atingir $\mathrm{pH}$ neutro e secagem a $100{ }^{\circ} \mathrm{C}$ durante $12 \mathrm{~h}$. Para estudar o processo de formação dos HDLs investigou-se o efeito da temperatura de síntese $\left(30\right.$ e $\left.45^{\circ} \mathrm{C}\right)$ e do $\mathrm{pH}$ final de síntese (9 e 12).

\section{Caracterização dos materiais}

A EAF teve a sua composição química e mineralógica determinada por fluorescência e difração de raios X. Os HDLs foram caracterizados por difração de raios X, método do pó - XRPD, espectrometria de energia dispersiva de raios X - EDS, microscopia eletrônica de varredura - MEV e espectroscopia vibracional na região do infravermelho - EIV. Os HDLs foram codificados com as iniciais da escória de alto forno (EAF) e do hidróxido duplo lamelar (HDL) e pelos valores de temperatura $(\mathrm{T})$ e $\mathrm{pH}$ de síntese $(\mathrm{pH})$, como: EAF-HDL-T-pH.

\section{Fluorescência de raios $X$}

A composição química da EAF foi determinada por fluorescência de raios X (FRX), utilizando-se um espectrômetro sequencial Axios Minerals, tubo de raios X cerâmico anodo de Rh de 2,4 kW, PANalytical. As concentrações dos elementos foram estabelecidas através do programa IQ+ Semiquant. A aquisição de dados foi feita com o software Super $Q$ Manager e o tratamento dos dados com o software $I Q+$, também da PANalytical, sendo o resultado normalizado para $100 \%$.

\section{Difração de raios $X$}

As análises mineralógicas foram realizadas por difratometria de raios X, método do pó (XRPD). Utilizou-se um difratômetro, marca PANalytical modelo X'PERT PRO MPD (PW 3040/60), com goniômetro PW 3050/60 (Theta/Theta), modelo PW 3373/00, foco fino longo, $2200 \mathrm{~W}, 60 \mathrm{Kv}$ e com tubo de raios X cerâmico de anodo de $\mathrm{Cu}\left(\mathrm{K} \alpha_{1} 1,540598 \AA\right)$. A aquisição de dados dos registros foi obtida com o software X'Pert Data Colletor, versão 2.1a, e o tratamento dos dados com o software $X^{\prime}$ Pert HighScore, versão 2.1b, também da PANalytical. Os registros foram realizados no intervalo de 5 a $75^{\circ} 2 \theta$.

\section{Microscopia eletrônica de varredura (MEV)}

A morfologia das partículas e o tamanho dos cristais foram determinados por microscopia eletrônica de varredura (MEV), usando um equipamento modelo LEO-1430. As condições de análises para imagens de elétrons secundários foram: corrente do feixe de elétrons de $90 \mu \mathrm{A}$, voltagem de aceleração constante de $10 \mathrm{Kv}$ e distância de trabalho de $12-15 \mathrm{~mm}$. As amostras foram previamente metalizadas com uma fina camada de ouro ou platina. Concomitante às análises de MEV, foi determinada a proporção entre cátions metálicos, mais especificamente $\mathrm{Mg}^{2+}: \mathrm{Al}^{3+}$, através da microanálise por espectrometria de dispersão de energia de raios X/EDS.

\section{Espectroscopia vibracional na região do infravermelho (EIV)}

O ânion carbonato intercalado na estrutura dos HDLs foi determinado por EIV. Os espectros vibracionais das amostras foram registrados no intervalo de 4000-400 cm-1, com resolução de $4 \mathrm{~cm}^{-1}$ e 16 varreduras. Os espectros foram registrados em um aparelho de marca Thermo Electron Corporation, modelo IR 100. As amostras foram secas ao ar em estufa a $100{ }^{\circ} \mathrm{C}$ por $24 \mathrm{~h}$ e, em seguida, colocadas em dessecador com sílica gel. Uma mistura de 200 mg de $\mathrm{KBr}$ grau espectroscópico e $1,5 \mathrm{mg}$ de cada amostra pulverizada foram homogeneizadas e preparadas em pastilhas de $1 \mathrm{~cm}$ de diâmetro a uma pressão de 1,8 Kbar sob vácuo.

\section{RESULTADOS E DISCUSSÃO}

\section{Escória de alto forno}

Os principias minerais observados no difratograma de raios $\mathrm{X}$ da EAF (Figura 1S, material suplementar) foram a gehlenita $\left(\mathrm{Ca}_{2} \mathrm{Al}\left[\mathrm{AlSiO}_{7}\right]\right)$ com reflexões 3,06, 2,84, 1,75 Å e a pseudowollastonita $\left(\mathrm{CaSiO}_{3}\right)$, com reflexões 3,23, 2,81, 1,98 ̊. Estes valores são semelhantes aos encontrados nas fichas PDF 01-087-0968 (gehlenita) e PDF 01-074-0874 (pseudowollastonita).

A composição química da EAF, expressa em óxido, está listada na Tabela 1. A análise química, por FRX, mostrou como constituintes principais $\mathrm{CaO}, \mathrm{SiO}_{2}, \mathrm{Al}_{2} \mathrm{O}_{3}$ e $\mathrm{MgO}$. Eles representam aproximadamente $98 \%$ da massa total da EAF e a razão $\mathrm{Mg} / \mathrm{Al}$ é 0,1 . A EAF é considerada ácida por apresentar a razão $\mathrm{CaO} / \mathrm{SiO}_{2}<1$. Os com- 
ponentes $\mathrm{Fe}_{2} \mathrm{O}_{3}, \mathrm{TiO}_{2}, \mathrm{MnO}, \mathrm{K}_{2} \mathrm{O}, \mathrm{Na}_{2} \mathrm{O}, \mathrm{P}_{2} \mathrm{O}_{5}$ e $\mathrm{SO}_{3}$ também estão presentes na $\mathrm{EAF}$, porém em pequenas quantidades.

Tabela 1. Composição química da EAF expressa em óxido

\begin{tabular}{cc}
\hline Constituintes & Concentração \%-massa \\
\hline $\mathrm{SiO}_{2}$ & 42,39 \\
$\mathrm{CaO}$ & 41,42 \\
$\mathrm{Al}_{2} \mathrm{O}_{3}$ & 13,32 \\
$\mathrm{MgO}$ & 1,07 \\
$\mathrm{Fe}_{2} \mathrm{O}_{3}$ & 0,40 \\
$\mathrm{~K}_{2} \mathrm{O}$ & 0,29 \\
$\mathrm{Na}_{2} \mathrm{O}$ & 0,18 \\
$\mathrm{MnO}$ & 0,44 \\
$\mathrm{P}_{2} \mathrm{O}_{5}$ & 0,01 \\
$\mathrm{SO}_{3}$ & 0,11 \\
$\mathrm{TiO}_{2}$ & 0,31 \\
$\mathrm{Total}$ & $\mathbf{9 9 , 9 4}$ \\
\hline
\end{tabular}

\section{Resíduo da digestão ácida da EAF}

O resíduo resultante da digestão da $\mathrm{EAF}$ em $\mathrm{HCl}$ foi analisado por meio de difração e fluorescência de raios X. Os padrões de difração do resíduo indicaram apenas a presença de substâncias amorfas. Sua composição química em base livre de umidade é fundamentalmente $\mathrm{SiO}_{2}$ (>99\%-massa) com apenas 0,24\%-massa de outros componetes $\left(\mathrm{CaO}, \mathrm{K}_{2} \mathrm{O}\right.$, e $\left.\mathrm{Al}_{2} \mathrm{O}_{3}\right)$. A concentração dos cátions (mmol) na solução de EAF é: $\mathrm{Ca}^{2+}=7,40, \mathrm{Al}^{3+}=2,61, \mathrm{Mg}^{2+}=0,27, \mathrm{Fe}^{\mathrm{n}+}=0,05, \mathrm{~K}^{+}=$ $0,06, \mathrm{Na}^{+}=0,06 \mathrm{e} \mathrm{Mn}^{\mathrm{n}+}=0,06(\mathrm{n}=2$ ou 3$)$.

\section{Hidróxidos duplos lamelares à base de EAF}

Os HDLs sintetizados em temperatura de $30^{\circ} \mathrm{C}$ (EAF-HDL-30-9 e EAF-HDL-30-12) mostram a intercalação do ânion carbonato $\left(\mathrm{CO}_{3}{ }^{2-}\right)$, formando, portanto, $\mathrm{HDLs}$ do sistema $\mathrm{Mg}-\mathrm{Al}-\mathrm{CO}_{3}$. A quantidade apropriada de $\mathrm{CO}_{3}{ }^{2-}$ para formação dos HDLs foi provavelmente introduzida através do sequestro de $\mathrm{CO}_{2}$ da atmosfera e da água deionizada, uma vez que no preparo das soluções de síntese não foi utilizada nenhuma substância contendo $\mathrm{CO}_{3}{ }^{2-}$.

Quando os HDLs são sintetizados à temperatura de $45^{\circ} \mathrm{C}(\mathrm{EAF}-$ -HDL-45-9 e EAF-HDL-45-12), além da presença do carbonato, verifica-se a intercalação do íon cloreto. $\mathrm{O}$ cloreto é proveniente tanto do $\mathrm{HCl}$, como do sal utilizado $\mathrm{MgCl}_{2} \cdot 6 \mathrm{H}_{2} \mathrm{O}$, adicionados no preparo da solução A. Portanto, os HDLs formados a $45^{\circ} \mathrm{C}$ são do sistema $\mathrm{Mg}-\mathrm{Al}-\mathrm{Cl}-\mathrm{CO}_{3}$. Miyata ${ }^{21}$ sintetizou vários compostos do tipo hidrotalcita e observou que o $\mathrm{CO}_{3}{ }^{2-}$ também estava presente em todos os sistemas, inclusive no $\mathrm{Mg}$-Al-Cl. A quantidade de $\mathrm{CO}_{3}{ }^{2-}$ contida nos HDLs foi incorporada nos produtos, porque o $\mathrm{CO}_{3}^{2-}$ é o ânion que melhor estabiliza a estrutura destes compostos e possui eletronegatividade maior que aquela observada nos ânions monovalentes.

A presença dos ânions foi determinada por análises de EDS (Tabela 2) e confirmada por espectroscopia vibracional na região do infravermelho. Para as amostras do sistema $\mathrm{Mg}-\mathrm{Al}-\mathrm{Cl}-\mathrm{CO}_{3}$, as análises por EDS indicam uma quantidade de cloreto entre 5,68 e 5,98\%-massa e o carbonato estava abaixo do limite de detecção do aparelho. Esse último, entretanto, foi confirmado através da análise de EIV. Nas amostras do sistema $\mathrm{Mg}-\mathrm{Al}-\mathrm{CO}_{3}, \mathrm{o} \mathrm{CO}_{3}{ }^{2-}$ pode ser confirmado tanto por espectroscopia vibracional na região do infravermelho como por EDS. Os valores observados estão entre 21,5 e 22,3\%-massa.
Tabela 2. Análise química semiquantitativa dos HDLs por EDS (\%-massa)

\begin{tabular}{ccccc}
\hline \multirow{2}{*}{ Elementos } & \multicolumn{4}{c}{ Amostra } \\
& $\begin{array}{c}\text { EAF- } \\
\text { HDL-30-9 }\end{array}$ & $\begin{array}{c}\text { EAF- } \\
\text { HDL-30-12 }\end{array}$ & $\begin{array}{c}\text { EAF- } \\
\text { HDL-45-9 }\end{array}$ & $\begin{array}{c}\text { EAF- } \\
\text { HDL-45-12 }\end{array}$ \\
\hline $\mathrm{C}$ & 4,46 & 4,31 & nd & nd \\
$\mathrm{O}$ & 63,10 & 61,01 & 54,88 & 55,88 \\
$\mathrm{Na}$ & 0,78 & 0,49 & 0,35 & 0,38 \\
$\mathrm{Mg}$ & 21,34 & 22,68 & 26,00 & 27,58 \\
$\mathrm{Al}$ & 8,79 & 6,86 & 11,40 & 9,07 \\
$\mathrm{Si}$ & 0,70 & 0,82 & 0,54 & 0,25 \\
$\mathrm{Cl}$ & 0,02 & 0,04 & 5,68 & 5,98 \\
$\mathrm{Ca}$ & 0,28 & 3,32 & nd & nd \\
$\mathrm{Ti}$ & 0,16 & 0,12 & 0,19 & 0,13 \\
$\mathrm{Mn}$ & 0,25 & 0,19 & 0,55 & 0,48 \\
$\mathrm{Fe}$ & 0,13 & 0,15 & 0,41 & 0,26 \\
\hline
\end{tabular}

nd - Não detectado.

Nos padrões de difração de raios $\mathrm{X}$ dos compostos sintetizados a 30 e a $45^{\circ} \mathrm{C}$, em pH $=9$ (Figura 1), observa-se que os HDLs EAF-HDL-30-9 e EAF-HDL-45-9 possuem espaçamentos basais $d(003)$ iguais a 7,73 e 7,81 $\AA$, respectivamente. As distâncias interplanares encontradas para os materiais sintetizados coincidem com os valores reportados na literatura para HDLs contendo carbonato $^{22}$ e cloreto. $^{23}$

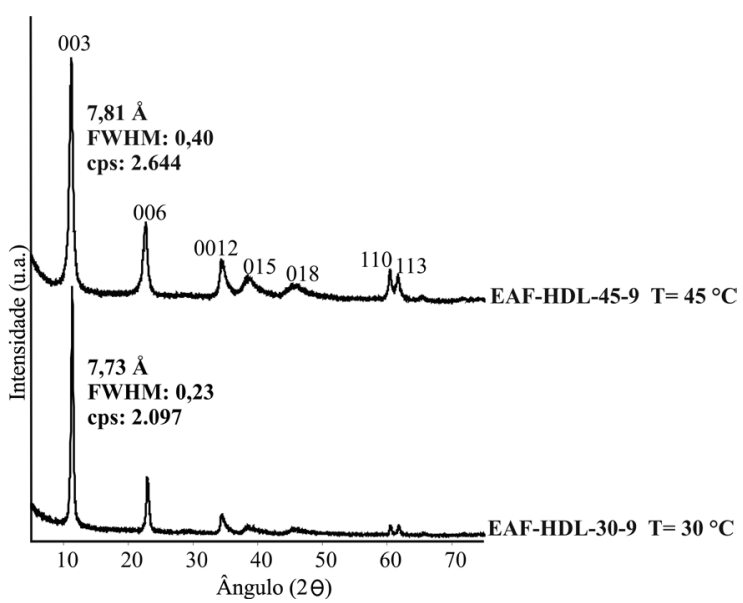

Figura 1. Difratogramas de raios $\mathrm{X}$ dos $\mathrm{HDLs}$ do sistema $\mathrm{Mg}-\mathrm{Al}-\mathrm{CO}_{3}$, sintetizado a $30^{\circ} \mathrm{C}$ (EAF-HDL-30-9) e do sistema $\mathrm{Mg}$-Al-Cl-CO , sintetizado a $45^{\circ} \mathrm{C}$ (EAF-HDL-45-9), preparados em $\mathrm{pH} 9$

A diferença entre os HDLs do sistema $\mathrm{Mg}-\mathrm{Al}-\mathrm{CO}_{3}$ e $\mathrm{Mg}$-Al-Cl$-\mathrm{CO}_{3}$ é demonstrada através dos valores de espaçamento basal e da largura a meia altura (FWHM) da reflexão (003). Na Figura 1 verifica-se que os HDLs do sistema $\mathrm{Mg}$ - $\mathrm{Al}-\mathrm{Cl}-\mathrm{CO}_{3}$ mostram maiores valores de $d$ e de FWHM =0,40. Segundo Miyata, ${ }^{21}$ quando dois ânions com pequena diferença entre seus raios estão presentes no mesmo espaço interlamelar, o padrão de difração desse composto mostrará uma refleção basal (003) mais intensa/larga, como se as duas reflexões basais dos dois sistemas de ânios isolados estivessem sobrepostas.

Os HDLs sintetizados a 30 e a $45^{\circ} \mathrm{C}$ foram obtidos em diferentes valores de $\mathrm{pH}$ final de síntese (9 e 12). Verifica-se, nas Tabelas $2 \mathrm{e}$ 3, que a razão molar entre $\mathrm{Mg}^{2+} / \mathrm{Al}^{3+}$, nos HDLs sintetizados, ficou entre 2,6 e 3,7, próximas da razão molar teórica da solução de síntese de partida $(3,5)$. Os HDLs preparados em elevado valor de $\mathrm{pH}$ mostraram razões molares $\mathrm{Mg} / \mathrm{Al}$ muito similares às razões molares 
teóricas. Este fato sugere que há uma correlação entre o teor de magnésio com o valor de $\mathrm{pH}$ final de síntese, além da influência da temperatura. Percebe-se uma tendência crescente da quantidade de $\mathrm{Mg}$ nos HDLs com o aumento do $\mathrm{pH}$ e da temperatura, o que pode indicar que nas condições de síntese, realizada em $\mathrm{pH}$ igual a 9, o magnésio continua solúvel, impossibilitando sua total precipitação. Cavani et al. ${ }^{20}$ ressaltam que cátions divalentes precipitam em valores de $\mathrm{pHs}$ mais elevados que os trivalentes.

A Tabela 2 mostra, ainda, a presença de outros elementos em menor percentagem. Estes apresentam comportamento contrário ao $\mathrm{Mg}$ em função do $\mathrm{pH}$. De modo geral, há uma redução na sua concentração com o aumento do $\mathrm{pH}$ de síntese. Isto sugere que, além do $\mathrm{Al}$, os cátions $\mathrm{Mn}^{\mathrm{n}+}$ e $\mathrm{Fe}^{\mathrm{n}+}(\mathrm{n}=2$ ou 3 ) foram provavelmente incorporados na lamela dos HDLs, ocupando alguns centros octaédricos. As espécies tetravalentes $\mathrm{Ti}^{4+}$ e $\mathrm{Si}^{4+}$ foram também observadas por Kuwahara et al. ${ }^{1}$ em seu trabalho sobre síntese de hidrocalumitas a partir de escória de alto forno. Segundo esses autores, estes elementos podem estar localizados sobre a superfície dos cristalitos dos HDL ou, ainda, podem formar oxiânions que são intercalados na estrutura dos HDLs, o que é corroborado por este trabalho.

Observa-se, ainda, que os HDLs preparados em baixo valor de pH têm menor razão molar $\mathrm{Mg} / \mathrm{Al}$ e, portanto, maior densidade de carga positiva nas suas lamelas, o que de certa forma favorece um menor grau de ordenamento estrutural e/ou redução do tamanho dos cristalitos, verificado através do alargamento das reflexões basais dos compostos EAF-HDL-45-9, FWHM = 0,40, contra EAF-HDL-45-12, $\mathrm{FWHM}=0,25$ (Figura 2). Portanto, os HDLs sintetizados em $\mathrm{pH}$ $=9$ apresentam menor grau de ordenamento estrutural que aqueles preparados em $\mathrm{pH}=12$. Estes resultados estão de acordo com as observações de Miyata $^{21}$ e Kuwahara et al., ${ }^{1}$ que mostraram que HDLs com diversos ânions intercalados e preparados em diferentes pH possuem a largura da reflexão (003) mais larga para cristalitos de menor tamanho, cujo pH de síntese também é menor.

Em todos os difratogramas de raios $\mathrm{X}$ dos compostos sintetizados, observam-se somente reflexões de estrutura lamelar, típica de hidrotalcita. Os resultados demonstram que os compostos apresentam pureza de fase e diferentes graus de ordenamento estrutural, com o maior ordenamento sendo obtido em $\mathrm{pH}=12$ (Figura 2). Percebe-se, também, que as intensidades dos picos se tornaram gradualmente mais intensas e agudas com o aumento do $\mathrm{pH}$ de síntese. Verificou-se que a intensidade do reflexo (003) do EAF-HDL-45-12, além de mais aguda, é mais intensa $(\mathrm{cps}=5.256)$ do que aquela observada no EAF-HDL-45-9 ( $\mathrm{cps}=2.644)$.

Comparando-se o perfil de difração de raios $\mathrm{X}$ dos compostos analisados, percebe-se o alto grau de pureza de fase dos HDLs sin-

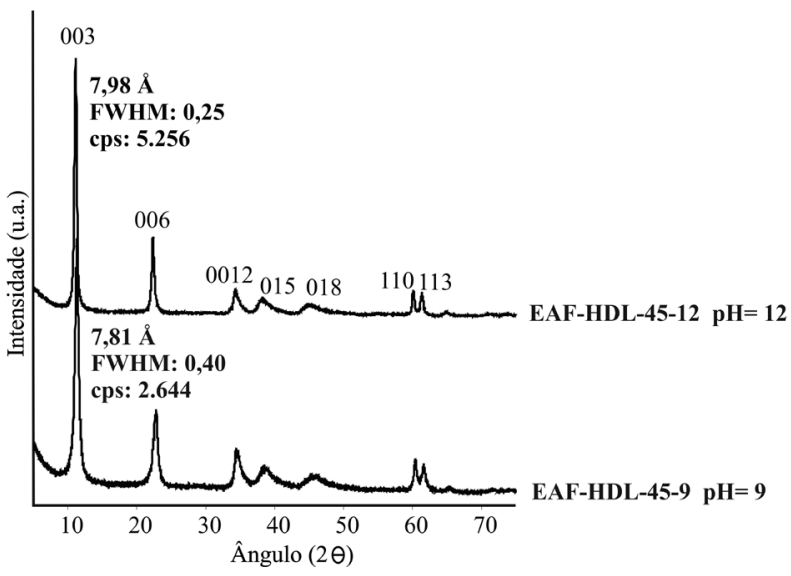

Figura 2. Difratogramas de raios $X$ dos $H D L$ s sintetizados a $45^{\circ} \mathrm{C}$ em $\mathrm{pH} 9$ (EAF-HDL-45-9) е pH 12 (EAF-HDL-45-12) tetizados a $\mathrm{pH}$ crescente, mesmo quando preparados em um grande intervalo de $\mathrm{pH}$ (9 a 12). A amostra EAF-HDL-30-12, sintetizada a $\mathrm{pH}=12$ e $30{ }^{\circ} \mathrm{C}$, foi a única que diferiu das demais. No seu difratograma verificou-se outra fase cristalina, com reflexões em 3,04; 2,29 e $2,10 \AA$, indicando a presença de calcita $\left(\mathrm{CaCO}_{3}\right)$ (Figura $2 \mathrm{~S}$, material suplementar). A formação da calcita foi, provavelmente, favorecida pelas condições de síntese e pelo alto teor em $\mathrm{CaO}$ (41,42\%-massa) na escória de alto forno. Estes resultados contradizem Cavani et $a l .,{ }^{20}$ que consideram difícil a obtenção de HDLs puros por meio da coprecipitação a $\mathrm{pH}$ crescente.

Os dados de distância interplanar $\mathrm{d}_{\mathrm{hkl}}$ dos HDLs sintetizados estão listados na Tabela 1S (material suplementar), juntamente com os dados da literatura. ${ }^{22,23}$

As reflexões $d_{h k l}$ observadas nos difratogramas dos HDLs sintetizados foram comparadas com os padrões do banco de dados do programa da Philips (X-Pert High Score), que se utiliza de padrões difratométricos do JCPDS-ICDD. Foi constatado que os valores concordam razoavelmente com os atribuídos à hidrotalcita $\left(\mathrm{Mg}-\mathrm{Al}-\mathrm{CO}_{3}\right)$, da ficha PDF (01-089-0460). Embora os HDLs EAF-HDL-45-9 e EAF-HDL-45-12 apresentem similaridade quando comparados com a ficha PDF de hidrotalcita, deve-se destacar que estes materiais apresentam, além do carbonato, o cloreto no seu espaço interlamelar.

Para uma análise estrutural mais precisa, os parâmetros cristalográficos da cela $a$ e $c$ foram calculados utilizando os dados de XRPD. O espaçamento basal $d$ foi calculado utilizando-se a Equação de Bragg, a partir da reflexão de maior ordem possível, neste caso (003). Os valores dos parâmetros $a$ e $c$ foram obtidos segundo as Equações propostas por Debye-Scherrer. ${ }^{22-26} \mathrm{O}$ parâmetro $c$ foi calculado através da multiplicação de $d(003)$ em Å pelo índice 3, ou seja, $c=3 d_{003}$. O parâmetro $a$ foi calculado por meio da multiplicação de $d(110)$ em Å por 2, isto é, $a=2 \mathrm{~d}_{110}$. Os resultados estão resumidos na Tabela 3, mostrando que os parâmetros estruturais concordam com os dados publicados para a hidrotalcita. ${ }^{27}$

Tabela 3. Efeito da temperatura e do $\mathrm{pH}$ de síntese sobre os parâmetros $a$ e $c$ dos HDLs

\begin{tabular}{lccccc}
\hline & \multirow{2}{*}{$\mathrm{pH}$} & \multicolumn{2}{c}{ Razão } & \multicolumn{2}{c}{ Parâmetros de cela $(\AA)$} \\
& $\left({ }^{\circ} \mathrm{C}\right)$ & final & molar Mg/Al & $a=b$ & $c$ \\
\hline EAF-HDL-30-9 & 30 & 9 & 2,7 & 3,06 & 23,18 \\
EAF-HDL-30-12 & 30 & 12 & 3,7 & 3,08 & 23,74 \\
EAF-HDL-45-9 & 45 & 9 & 2,6 & 3,06 & 23,43 \\
EAF-HDL-45-12 & 45 & 12 & 3,4 & 3,08 & 23,95 \\
\hline
\end{tabular}

Analisando a Tabela 3 é possível observar um aumento nos valores dos parâmetros $a$ e $c$ da cela unitária com o aumento dos valores da temperatura e do $\mathrm{pH}$ final de síntese. Os maiores valores de $a$ e $c$ observados comprovam que os HDLs sintetizados nestas condições apresentam um maior teor em $\mathrm{Mg}^{2+}$, cujo raio iônico é maior que o do $\mathrm{Al}^{3+}$. $\mathrm{O}$ aumento do parâmetro $c$ pode ser explicado pela redução da força de atração eletrostática entre as lamelas e as espécies interlamelares, já que $\mathrm{Mg}^{2+}$ possui uma menor densidade de carga que o $\mathrm{Al}^{3+} .^{28}$

A morfologia e o tamanho de partícula dos cristais dos HDLs sintetizados foram observados por microscopia eletrônica de varredura (MEV). A imagem de MEV do composto EAF-HDL-30-12 sintetizado a $30{ }^{\circ} \mathrm{C} \mathrm{em} \mathrm{pH}=12$ pode ser vista na Figura 3. Nas micrografias das amostras, verifica-se uma morfologia homogênea, com cristalitos de tamanhos semelhantes, com hábito hexagonal, não muito bem desenvolvidos. Estes cristalitos estão aglomerados, mostrando nitidamente uma microporosidade. Na amostra EAF-HDL-30-12, além dos cristalitos de HDLs, observa-se a presença de romboédros 
de calcita (Figura 3S, material suplementar). Os HDLs com cristalitos de maior tamanho foram aqueles preparados em condições de síntese onde os valores de $\mathrm{pH}$ foram mais elevados.

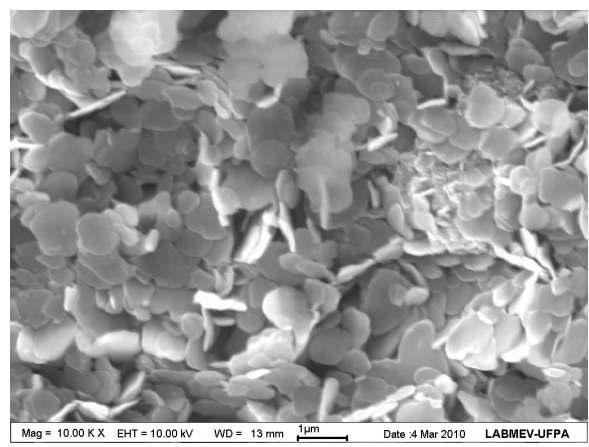

Figura 3. Imagem de $M E V$ do composto EAF-HDL-30-12 sintetizado a 30 ${ }^{\circ} \mathrm{C}$ em $\mathrm{pH} 12$

Os resultados da análise dos HDLS por espectroscopia vibracional na região do infravermelho mostram bandas semelhantes àquelas observadas na hidrotalcita. ${ }^{29} \mathrm{Na}$ Figura 4 observa-se a sobreposição dos espectros vibracionais na região do infravermelho dos HDLs do sistema Mg-Al-Cl- $\mathrm{CO}_{3}$, sintetizados a $45^{\circ} \mathrm{C}$ em pH 9 (EAF-HDL-45-9) e 12 (EAF-HDL-45-12). Observa-se uma banda larga e intensa que absorve a $3477-3577 \mathrm{~cm}^{-1}$, presente em todos os espectros, aqui atribuída às vibrações de estiramento da ligação $\mathrm{O}-\mathrm{H}$ das moléculas de água extrínsecas (fisissorvidas na lamela), intrínsecas (interlamelares) e, também, dos grupos hidroxila das lamelas. A presença de um ombro próximo a $3000 \mathrm{~cm}^{-1}$, comum nos espectros dos HDLs do sistema $\mathrm{Mg}-\mathrm{Al}-\mathrm{CO}_{3}$, é típico de ligações de hidrogênio entre a água e o ânion carbonato. Há uma pequena banda comum aos espectros de HDLs do sistema Mg-Al-CO ${ }_{3}$ EAF-HDL-30-9 e EAF-HDL-30-12 (1645 $\left.\mathrm{cm}^{-1}\right)$ e do sistema Mg-Al-Cl-CO 3 EAF-HDL-45-9 $\left(1633 \mathrm{~cm}^{-1}\right)$ e EAF-HDL-45-12 (1634 cm-1), atribuída à deformação angular H-O-H das moléculas de água. Nos espectros vibracionais dos HDLs dos dois sistemas $\mathrm{Mg}-\mathrm{Al}-\mathrm{CO}_{3}$ e $\mathrm{Mg}-\mathrm{Al}-\mathrm{Cl}-\mathrm{CO}_{3}$ foram observadas três bandas de absorções ativas: $1369-1455,850-880$ e $615-665 \mathrm{~cm}^{-1}$, indicativas da presença do ânion carbonato em um ambiente simétrico, como no caso do $\mathrm{CO}_{3}{ }^{2-}$ livre. Verifica-se em todos os espectros a existência de uma banda bastante intensa a $1369 \mathrm{~cm}^{-1}$ (EAF-HDL-30-9) e $1455 \mathrm{~cm}^{-1}$ (EAF-HDL-30-12), nos HDLs do sistema Mg-Al-CO ${ }_{3}$, e bem menos intensa a $1370 \mathrm{~cm}^{-1}$ (EAF-HDL-45-9) e $1386 \mathrm{~cm}^{-1}$ (EAF-HDL-45-12) nos HDLs do sistema $\mathrm{Mg}-\mathrm{Al}-\mathrm{Cl}-\mathrm{CO}_{3}$, que está relacionada às vibrações de estiramento $\mathrm{C}-\mathrm{O}$ dos grupos $\mathrm{CO}_{3}{ }^{2-}$. Em torno de 1400 $\mathrm{cm}^{-1}$, observa-se a presença de um ombro, principalmente nos HDLs do sistema $\mathrm{Mg}-\mathrm{Al}-\mathrm{CO}_{3}$ que, segundo Miyata, ${ }^{22}$ pode estar relacionado com o abaixamento da simetria do carbonato e à desordem no espaço interlamelar. Na região entre $850-880 \mathrm{~cm}^{-1}$, observa-se um ombro em todos os espectros apresentados, com aparecimento de uma banda fina a $877 \mathrm{~cm}^{-1}$ no espectro da amostra EAF-HDL-30-12. Em baixas frequências, a $615-665 \mathrm{~cm}^{-1}$ aparece uma banda larga, encontrada em todos os espectros, que é típica da deformação metal-oxigênio (M-O) das lamelas do HDL, possivelmente do óxido de magnésio e alumínio.

De modo geral, os perfis dos espectros vibracionais dos HDLs dos sistemas $\mathrm{Mg}-\mathrm{Al}-\mathrm{CO}_{3}$ e $\mathrm{Mg}-\mathrm{Al}-\mathrm{Cl}-\mathrm{CO}_{3}$ são semelhantes. A diferença entre os perfis está na região entre 1369 a $1455 \mathrm{~cm}^{-1}$, onde a intensidade da banda correspondente às vibrações de estiramento $\mathrm{C}-\mathrm{O}$ do grupo $\mathrm{CO}_{3}{ }^{2-}$ é menor para os $\mathrm{HDLs}$ do sistema $\mathrm{Mg}-\mathrm{Al}-\mathrm{Cl}-\mathrm{CO}_{3}$, sugerindo que os íons cloreto substituem parcialmente os carbonatos. Pela intensidade das bandas nesta região, pode-se dizer que o íon cloreto, além de estar presente no espaço interlamelar dos HDLs, sintetizados a $45^{\circ} \mathrm{C}$, está em maior quantidade nos HDLs preparados em altos

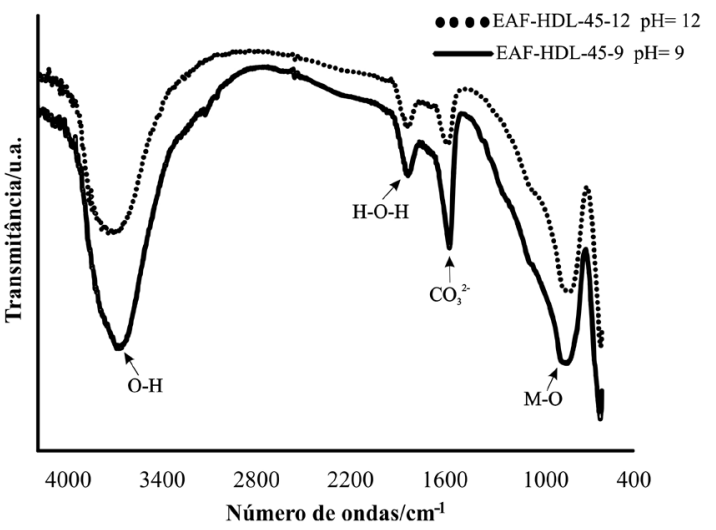

Figura 4. Sobreposição dos espectros vibracionais na região do IV dos HDLs do sistema $\mathrm{Mg}$ - $\mathrm{Al}$-Cl-CO , sintetizados a $45^{\circ} \mathrm{C}$ em pH 9 (EAF-HDL-45-9) e pH 12 (EAF-HDL-45-12)

valores de $\mathrm{pH}$, como verificado pela menor intensidade desta banda para a amostra EAF-HDL-45-12 (Figura 4).

\section{CONCLUSÕES}

As informações aqui apresentadas mostram que é possível preparar uma variedade de hidróxidos duplos lamelares, com estruturas semelhantes à da hidrotalcita, utilizando-se EAF como matéria-prima. HDLs com elevada pureza de fase e grau de ordenamento estrutural foram sintetizados por meio da coprecipitação a $\mathrm{pH}$ crescente.

Os HDLs formados pertencem aos sistemas $\mathrm{Mg}-\mathrm{Al}-\mathrm{CO}_{3}$ e $\mathrm{Mg}-$ -Al-Cl- $\mathrm{CO}_{3}$. As análises por meio de DRX revelaram que os HDLs sintetizados a $30{ }^{\circ} \mathrm{C}$ em pH 9 e 12 são do sistema $\mathrm{Mg}-\mathrm{Al}-\mathrm{CO}_{3}$. A calcita foi observada somente quando o $\mathrm{pH}$ de síntese foi 12 , estando ausente em valores mais baixos. A quantidade necessária de carbonato, nas soluções de síntese, para formação dos HDLs foi capturada da atmosfera e/ou da água utilizada. Nos difratogramas das amostras sintetizadas a $45^{\circ} \mathrm{C}$, em pH 9 e 12 , foram observados somente reflexos típicos de hidrotalcita e os HDLs são do sistema $\mathrm{Mg}-\mathrm{Al}-\mathrm{Cl}-\mathrm{CO}_{3}$. Os íons cloreto intercalados nos HDLs se originaram do $\mathrm{HCl}$ e $\mathrm{MgCl}_{2} \cdot 6 \mathrm{H}_{2} \mathrm{O}$, usados no preparo das soluções de síntese.

Os HDLs sintetizados nos dois sistemas, quando preparados em $\mathrm{pH} 12$, apresentaram-se com maior grau de ordem estrutural e com maiores valores de $d$ e dos parâmetros $a$ e $c$ da cela unitária.

A razão molar $\mathrm{Mg} / \mathrm{Al}$ nos HDLs foi influenciada pelo $\mathrm{pH}$ e temperatura de síntese. Os HDLs preparados em valores de $\mathrm{pH}$ e temperatura mais elevados mostraram razão molar $\mathrm{Mg} / \mathrm{Al}$ próxima dos valores da solução de síntese. Os outros cátions metálicos (Mn, $\mathrm{Fe}, \mathrm{Ti}, \mathrm{Si}$ ), que ocorrem em menor percentagem, estão provavelmente inclusos na estrutura dos HDLs. De modo geral, a quantidade destes cátions foi maior nas amostras preparadas a $45^{\circ} \mathrm{C}$ e em pH 9.

As análises de EIV confirmaram que em todos os espectros vibracionais há a presença de bandas características do ânion carbonato, com maior diferença na intensidade da banda na região entre $1369-1455 \mathrm{~cm}^{-1}$. Nos espectros vibracionais dos HDLs do sistema $\mathrm{Mg}-\mathrm{Al}-\mathrm{Cl}-\mathrm{CO}_{3}$, a intensidade da banda nessa região é bem menos intensa que aquela observada nos HDLs do sistema $\mathrm{Mg}-\mathrm{Al}-\mathrm{CO}_{3}$. Isso confirma a presença do íon cloreto substituindo parcialmente o carbonato no espaço interlamelar.

Os cristalitos de HDLs dos dois sistemas apresentam hábito hexagonal e formam aglomerados que desenvolvem uma microporosidade.

Deve-se ressaltar que, apesar de haver relatos de síntese de HDLs a partir de escórias, este é o primeiro relato de processo de síntese de HDLs à base de escória de alto forno, com estruturas semelhantes à da hidrotalcita. 


\section{MATERIAL SUPLEMENTAR}

A Figura 1S mostra a composição mineralógica da EAF; a Figura $2 \mathrm{~S}$ apresenta os difratogramas das amostras sintetizadas a $30{ }^{\circ} \mathrm{C}$ em pH 9 (EAF-HDL-30-9) e pH 12 (EAF-HDL-30-12). A Figura $3 \mathrm{~S}$ apresenta uma micrografia destacando os romboedros de calcita da amostra EAF-HDL-30-12. Apresenta-se também a Tabela $1 \mathrm{~S}$, que mostra os dados de distância interplanar $\mathrm{d}_{\mathrm{hkl}}$ dos HDLs sintetizados.. Esse material está disponível em http://quimicanova.sbq.org.br na forma de arquivo PDF, com acesso livre.

\section{AGRADECIMENTOS}

Ao CNPq pela bolsa de doutorado de M. M. Rebelo e à CAPES e ao Programa de Pós- Graduação em Geologia e Geoquímica pelo apoio financeiro e uso dos laboratórios de Análises Químicas, Difração de raios X, Fluorescência de raios X, MEV e EIV. Os autores gostariam ainda de fazer um agradecimento especial ao Mc. N. Valente pelo apoio no laboratório de análises químicas do PPGG-UFPA.

\section{REFERÊNCIAS}

1. Kuwahara,Y.; Ohmichi,T.; Kamegawa, T.; Mori, K.; Yamashita, H.; J. Mater. Chem. 2010, 20, 5052.

2. http://www.acobrasil.org.br, acessada em Dezembro 2011.

3. Velten, R. Z.; Sant'Ana, A. P.; de Lima, D. C.; Silva, C. H. C.; de Carvalho, C. A. B.; Couto, L. G.; Machado, C. C.; Revista Árvore 2006, $30,235$.

4. Batagin, A. F.; Esper, M. W.; Contribuição ao conhecimento das propriedades do cimento Portland de alto-forno, $\mathrm{ABCP}$ : São Paulo, 1988.

5. Cincotto, M. A.; Batagin, A. F.; Agopyan, V.; Caracterização da escória granulada de alto-forno e seu emprego como aglomerante e agregado, IPT, Boletim 65: São Paulo, 1992.

6. Mendes, W.; Dissertação de Mestrado, Universidade Estadual de Campinas, Brasil, 1999.
7. Soares, A. K.; Caracterização de escórias siderúrgicas com propriedades aglomerantes, ABCP: São Paulo, 1982.

8. Murphy, J. N.; Maadowcroft, T. R.; Barr, P. V.; Can. Metall. Q. 1997, 36,315 .

9. Shi, C.; Qian, J.; Resources, Conservation and Recycling 2000, 29, 195.

10. Allmann, R.; Chimia 1970, 24, 99.

11. Taylor, H. F. W.; Mineral. Mag. 1973, 39, 377.

12. Bish, D. L.; Bulletin Mineralogie 1980, 103, 170.

13. Silva, D. de O.; Constantino, V. R. L.; Gordijo, C. R.; Br PI 0700770-1 A, 2008.

14. Manju, G. N.; Gigi, M. C.; Anirudhan, T. S.; Indian J. Chem. Technol. 1999, 6,134.

15. Cunha, V. R. R.; Ferreira, A. M. da C.; Constantino, V. R. L.; Tronto, J.; Valim, J. B.; Quim. Nova 2010, 33, 159.

16. Wypych, F.; Br PI 0702235-2 A2, 2009.

17. Queiroz, R. M.; Pires, L. H. O.; Souza, R. C. P.; Zamian, J. R.; Souza, A. G.; Rocha Filho, G. N.; Costa, C. E. F.; J. Therm. Anal. Calorim. 2009, 97, 163.

18. Silva, C. C. C. M.; Ribeiro, N. F. P.; Souza, M. M. V. M.; Aranda, D. A. G.; Fuel Process. Technol. 2010, 91, 205.

19. Hayaji, S.; Norihiro, M.; Hideki, Y.; Jp 2006-151744, 2006.

20. Cavani, F.; Trifiró, F.; Vaccari, A.; Catal. Today 1991, 11, 177.

21. Miyata, S.; Clays Clay Miner. 1983, 31, 305.

22. Ross, G. J.; Kodama, H.; Am. Mineral. 1967, 52, 1036.

23. Miyata, S.; Clays Clay Miner. 1975, 23, 369.

24. Allmann, R.; Acta Crystallogr., Sect. B: Struct. Sci. 1968, 24, 972.

25. El Malki, K.; Roy, A. de; Besse, J. P.; Nanostructured Materials 1993, $2,167$.

26. Ku trowski, P.; Sułkowska, D.; Chmielarz, L.; Rafalska-Łasocha, A.; Dudek, B.; Dziembaj, R.; Microporous Mesoporous Mater. 2005, 78, 11.

27. Vaccari, A.; Catal. Today 1998, 41, 53.

28. Han, S.; Hou, W.; Zhang, C.; Sun, D.; Huang, X.; Wang, G.; J. Chem. Soc. Trans. Faraday 1998, 94, 915

29. Andrade, R. de S. dos S.; Dissertação de Mestrado, Universidade Estadual de Campinas, Brasil, 1997. 